\title{
Inhaltsverzeichnis Band 2: \\ Betriebsmittel und ihre quasistationäre Modellierung
}

\author{
Inhaltsverzeichnis Band 1: \\ Grundlagen, Systemaufbau und Methoden $-\mathrm{X}$ \\ Inhaltsverzeichnis Band 3: \\ Systemverhalten und Berechnung von Drehstromsystemen - XV \\ Größenbezeichnungen $-\mathbf{X X I}$
}

$1 \quad$ Einführung und Übersicht -1

2 Synchronmaschinen - 3

2.1 Prinzipieller Aufbau einer Synchronmaschine und Wicklungsschema - 3

2.1.1 Ständerwicklungen und Ständerdrehfeld -3

2.1.2 Läuferwicklung und Läuferdrehfeld - 11

2.1.3 Wicklungsschema und Zweiachsentheorie - 14

2.2 Nichtstationäres Betriebsverhalten — 20

2.3 Quasistationäres Modell - 21

2.4 Ersatzschaltungen für die Symmetrischen Komponenten - 24

2.4.1 Ersatzschaltungen für das Mitsystem - 25

2.4.2 Ersatzschaltung für das Gegensystem - 33

2.4.3 Ersatzschaltung für das Nullsystem - 34

$2.5 \quad$ Funktionsweise und stationäres Betriebsverhalten - 35

2.5.1 Funktionsweise - 35

2.5.2 Stromquellenersatzschaltung für den stationären Zustand - 36

2.5.3 Leerlauf und Polradspannung - 37

2.5.4 Ankerrückwirkung - 37

2.6 Stationäres Betriebsverhalten und Zeigerbilder - 40

2.6.1 Blindleistungsregelung $-\mathbf{4 0}$

2.6.2 Wirkleistungsregelung - 41

2.6.3 Zeigerbild der Vollpolsynchronmaschine - 43

2.6.4 Zeigerbild der Schenkelpolsynchronmaschine - 44

2.7 Leistung und Drehmoment - 44

2.7.1 Leistungsfluss in einer Drehfeldmaschine - $\mathbf{4 5}$

2.7.2 Drehmoment und Wirkungsgrad einer Drehfeldmaschine - 46

2.7.3 Leistungsfluss, Wirkungsgrad und Drehmoment einer Synchronmaschine - 47

2.7.4 Vom Synchrongenerator an das Netz abgegebene Leistung — 47 
2.7.5 Wirkleistung-Winkel-Kennlinie - $\mathbf{4 8}$

2.7.6 Blindleistung-Winkel-Kennlinie - $\mathbf{5 0}$

2.7.7 Leistungsdiagramm $\mathbf{- 5 1}$

2.8 Bewegungsgleichung - 53

2.9 Blockgröße und Bemessungsgrößen von Turbogeneratoren - 56

2.10 Erregersysteme von Synchronmaschinen - $\mathbf{5 7}$

3 Asynchronmaschinen - 65

3.1 Aufbau und Betriebsweise -65

3.1.1 Kurzschlussläufer - 65

3.1.2 Schleifringläufer -66

3.2 Wirkungsprinzip und Betriebsweise - 67

3.3 Ersatzschaltungen für die Symmetrischen Komponenten - 69

3.3.1 Ersatzschaltungen für das Mitsystem - 70

3.3.2 Ersatzschaltung für das Gegensystem - 72

3.3.3 Ersatzschaltung für das Nullsystem - 73

3.4 Bestimmung der Elemente der vereinfachten Ersatzschaltung — 74

3.5 Leistungsfluss und Drehmoment - 74

3.6 Bewegungsgleichung -77

3.7 Zeigerbild $\longrightarrow \mathbf{7 8}$

$4 \quad$ Ersatznetze -79

4.1 Ersatzschaltung für das Mitsystem - 79

4.2 Ersatzschaltung für das Gegensystem $-\mathbf{8 0}$

4.3 Ersatzschaltung für das Nullsystem $-\mathbf{8 0}$

5 Transformatoren -83

5.1 Bauarten und Einsatz von

Wechsel- und Drehstromtransformatoren - 83

5.1.1 Kernbauarten von Wechsel- und Drehstromtransformatoren - 83

5.1.2 Wicklungen, Kühlung und Bemessungsgrößen von Drehstromtransformatoren $-\mathbf{8 4}$

5.2 Einphasentransformator $-\mathbf{8 7}$

5.2.1 Strom- und Spannungsgleichung und Flussverteilung $-\mathbf{8 7}$

5.2.2 Ersatzschaltung des Einphasentransformators - 89

5.2.3 Vereinfachte Ersatzschaltung eines Einphasentransformators - $\mathbf{9 0}$

5.2.4 Idealer Transformator - 91

5.3 Drehstromtransformatoren - 91

5.3.1 Schaltungen von Drehstromwicklungen - 91

5.3.2 Schaltgruppen von Drehstromtransformatoren - 94

5.3.3 Übersetzungsverhältnis von Drehstromtransformatoren — 96 
5.4 Einsatz von Drehstromtransformatoren - 99

5.4.1 Maschinen- oder Blocktransformatoren - $\mathbf{1 0 0}$

5.4.2 Blockeigenbedarfstransformatoren $-\mathbf{1 0 0}$

5.4.3 Netzkuppeltransformatoren - 101

5.4.4 Verteilungstransformatoren $-\mathbf{1 0 2}$

5.4.5 Ortsnetztransformatoren - $\mathbf{1 0 2}$

5.5 Ersatzschaltungen für die Symmetrischen Komponenten - 104

5.5.1 Ersatzschaltung für das Mitsystem - 104

5.5.2 Ersatzschaltung für das Gegensystem - 105

5.5.3 Ersatzschaltung für das Nullsystem - 106

5.6 Bestimmung der Ersatzschaltungselemente - $\mathbf{1 0 8}$

5.6.1 Kurzschlussversuch und relative

Bemessungskurzschlussspannung - 108

5.6.2 Leerlaufversuch - $\mathbf{1 1 0}$

5.6.3 Bestimmung der Nullsystemgrößen - 112

5.7 Betriebsverhalten - 112

5.7.1 Spannungsabfall und Kapp'sches Dreieck — 112

5.7.2 Leerlauf - $\mathbf{1 1 4}$

5.7.3 Kurzschluss - 115

5.7.4 Wirkleistungsverluste und Blindleistungsbedarf -116

5.7.5 Wirkungsgrad - 117

5.8 Unsymmetrische Belastung und Sternpunktbelastbarkeit - 120

5.8.1 Durchflutungsgleichgewicht -120

5.8.2 Sternpunktbelastbarkeit Yyn0-Transformator mit Drei- und Fünfschenkelkern - 121

5.8.3 Sternpunktbelastbarkeit Yyn0d5-Transformator mit Drei- und Fünfschenkelkern — 126

5.8.4 Sternpunktbelastbarkeit Dyn5-Transformator mit Drei- und Fünfschenkelkern — 127

5.8.5 Sternpunktbelastbarkeit Yzn5-Transformator mit Drei- und Fünfschenkelkern - 130

5.9 Dreiwicklungstransformator - $\mathbf{1 3 2}$

5.10 Parallelbetrieb von Transformatoren - 135

5.11 Spartransformator - $\mathbf{1 3 8}$

5.11.1 Typ- und Durchgangsleistung - 139

5.11.2 Ersatzschaltung für das Mitsystem - $\mathbf{1 4 0}$

5.11.3 Relative Bemessungskurzschlussspannung - $\mathbf{1 4 2}$

5.12 Regeltransformator - $\mathbf{1 4 4}$

5.12.1 Längsregelung - $\mathbf{1 4 5}$

5.12.2 Querregelung - 145

5.12.3 Schrägregelung - 146 
6 Leitungen: Freileitungen und Kabel - 147

6.1 Übersicht -147

6.2 Drehstrom-Freileitung - $\mathbf{1 5 0}$

6.2.1 Aufbau von Freileitungen -151

6.2.2 Maste - 153

6.2.3 Leiterseile - 156

6.2.4 Erdseil -162

6.2.5 Isolatoren und Armaturen - 163

6.2.6 Mastfundament und bauliche Maßnahmen — 164

6.2.7 Querung von Verkehrswegen, Gewässern und Waldgebieten — 169

6.3 Drehstromkabel - $\mathbf{1 7 0}$

6.3.1 Übersicht 170

6.3.2 Aufbau von Energiekabeln und Aufbauelemente - 171

6.3.3 Kabeltransport und Kabellegung - 187

6.3.4 Querung von Verkehrswegen — 193

6.3.5 Kabelhochspannungsprüfung - 194

6.4 Leitungsgleichungen im Frequenzbereich - 195

6.4.1 Lösung der Leitungsgleichungen, Wellenimpedanz und Ausbreitungskonstante - 195

6.4.2 Sonderfall der verlustlosen Leitung - 196

6.4.3 Sonderfall der verlustarmen Leitung —-197

6.5 Leitungsparameter - 198

6.5.1 Ohmsch-induktive Kopplung - 198

6.5.2 Kapazitive Kopplung - 203

6.5.3 Verdrillung - 206

6.5.4 Typische Parameter von Freileitungen und Kabel - 213

6.6 Vierpolgleichungen und Ersatzschaltungen - 214

6.6.1 Kettenform - 214

6.6.2 Admittanzform und ח-Ersatzschaltung - 215

6.6.3 Impedanzform und T-Ersatzschaltung - $\mathbf{2 1 5}$

6.6.4 Ersatzschaltungen für die elektrisch kurze Leitung - 216

6.6.5 Vereinfachte Ersatzschaltung — 217

6.7 Betriebsverhalten - 217

6.7.1 Zeigerbild und Spannungsabfall -217

6.7.2 Übertragbare Leistung - 220

6.7.3 Verluste und Blindleistungsbedarf -221

6.7.4 Natürlicher Betrieb (Anpassung) - 222

6.7.5 Leerlaufende Leitung, Ladestrom und Ferranti-Effekt -225

6.7.6 Kurzgeschlossene Leitung - 227 
7 Drosselspulen, Kondensatoren und Kompensation - 229

7.1 Reihendrosselspule zur Begrenzung von Kurzschlussströmen - 229

7.2 Paralleldrosselspule zur Ladestromkompensation - 230

7.3 Sternpunktdrosselspule zur Sternpunkterdung - 233

7.4 Reihenkondensator zur Spannungsund Stabilitätsverbesserung - 234

7.4.1 Einsatz im Mittelspannungsnetz zur Spannungsbetragsverbesserung — 235

7.4.2 Einsatz im Höchstspannungsnetz zur Stabilitätsverbesserung — 236

7.5 Parallelkondensatoren -237

A Anhang - 241

A.1 Ausgewählte SI-Basis-Einheiten - 241

A.2 Ausgewählte abgeleitete SI-Einheiten — 241

A.3 Naturkonstanten und mathematische Konstanten -242

Literaturverzeichnis -243

Stichwortverzeichnis -245 
